\title{
Management of hypotrichosis of the eyelashes: Focus on bimatoprost
}

\author{
Steven Fagien \\ Aesthetic Eyelid Plastic Surgery, \\ Boca Raton, FL, USA
}

This article was published in the following Dove Press journal:

Clinical, Cosmetic and Investigational Dermatology

II April 2010

Number of times this article has been viewed

Correspondence: Steven Fagien 660 Glades Road, Suite 210, Boca Raton, FL 33431, USA

$\mathrm{Tel}+\mid$ 56I 3939898

$\mathrm{Fax}+|56| 3470772$

Email sfeyeplastixl@aol.com

\begin{abstract}
Prominent eyelashes are generally recognized as enhancing beauty and are often desired by women. Until recently, the options available to augment the prominence of eyelashes were limited to makeup, over-the-counter products, artificial eyelashes, and eyelash transplantation. Originally approved for the treatment of ocular hypertension, the prostamide, bimatoprost, is now approved for the treatment of hypotrichosis of the eyelashes. Bimatoprost ophthalmic solution $0.03 \%$, applied once daily to the skin of the upper eyelid margin using sterile singleuse-per-eye applicators, increases eyelash growth, including length, thickness, and darkness. The effectiveness of bimatoprost for eyelash growth has been demonstrated by clinician ratings, digital image analysis, and patient-reported measures of satisfaction. The effects of bimatoprost treatment on eyelash length, thickness, and darkness are believed to result from longer anagen duration, increased hair bulb thickness, and increased melanogenesis, respectively. Dermally applied bimatoprost appears to be associated with a lower incidence of adverse events than administration of the medication as an eyedrop. This more favorable safety and tolerability profile is likely mediated by decreased exposure of ocular tissues to bimatoprost when applied dermally. Taken together, available data suggest that cutaneous application of bimatoprost ophthalmic solution $0.03 \%$ safely and effectively enhances upper eyelash growth.
\end{abstract}

Keywords: hypotrichosis, eyelashes, bimatoprost, hair follicle, prostamide

\section{Introduction}

Although the ideals of physical beauty vary over time and by culture, general consensus supports the integral role of the eyes in defining beauty. ${ }^{1}$ The practice of taking steps to enhance the appearance of the eyes, specifically the eyelids and eyelashes, dates back thousands of years. ${ }^{2}$ Mulhern et al showed that enhancing the appearance of women's eyes (using eyeliner, eye shadow, and mascara) significantly increased attractiveness as rated by both male and female observers. ${ }^{3}$ With respect to women's eyelashes, most cultures highly value long thick lashes, and an increase in eyelash growth is thought to confer a positive psychologic effect. ${ }^{4-6}$

Several options exist for women looking to enhance the appearance of their eyelashes. Some agents, such as mascara and artificial eyelashes, have been available for decades. Only recently, however, has the US Food and Drug Administration (FDA) approved a product, bimatoprost ophthalmic solution $0.03 \%$ (Latisse $^{\mathbb{B}}$; Allergan, Inc., Irvine, CA), for the treatment of hypotrichosis of the eyelashes. This product has demonstrated an increase in the growth of eyelashes, including length, thickness, and darkness. ${ }^{7}$ This article will review the currently available options to improve the appearance of eyelashes, with a focus on the recently approved prescription drug, 
bimatoprost ophthalmic solution $0.03 \%$. To better understand the mechanisms of action by which bimatoprost ophthalmic solution $0.03 \%$ is believed to influence eyelash growth, we will also review the physiology of normal eyelash growth. Finally, the clinical data on the efficacy of bimatoprost for eyelash growth and the agent's safety data, both as treatment for hypotrichosis (applied dermally) and ocular hypertension (instilled ocularly), will be discussed.

\section{Eyelash physiology}

Physiologically, eyelashes serve a protective function and, as with all hairs on the body, are generated by the continuous cycling of hair follicles. They prevent debris from entering the eye and are responsible for triggering the blink reflex when touched. ${ }^{8-10}$ Similar to other hair follicles on the body, eyelash follicles are connected to sebaceous glands (ie, glands of Zeis). ${ }^{9,10}$ Unlike other follicles, however, the follicles of eyelashes are not associated with arrectores pilorum. ${ }^{11}$ The number of follicles cannot be increased after birth because all follicles develop during embryogenesis. ${ }^{12}$ Individuals can experience the loss of previously normal eyelashes, which may or may not be accompanied by the destruction of their respective hair follicles. This condition, madrosis (also referred to as milphosis), can have numerous causes, including alopecia areata, infection (eg, leprosy), endocrine disease (eg, hypothyroidism), medications (eg, antimetabolites), radiation, or trauma. ${ }^{10}$

As a result of their length, medullation, and dark pigmentation, eyelashes are properly classified as terminal hairs, in contrast with vellus or intermediate hairs. ${ }^{11}$ In fact, they represent

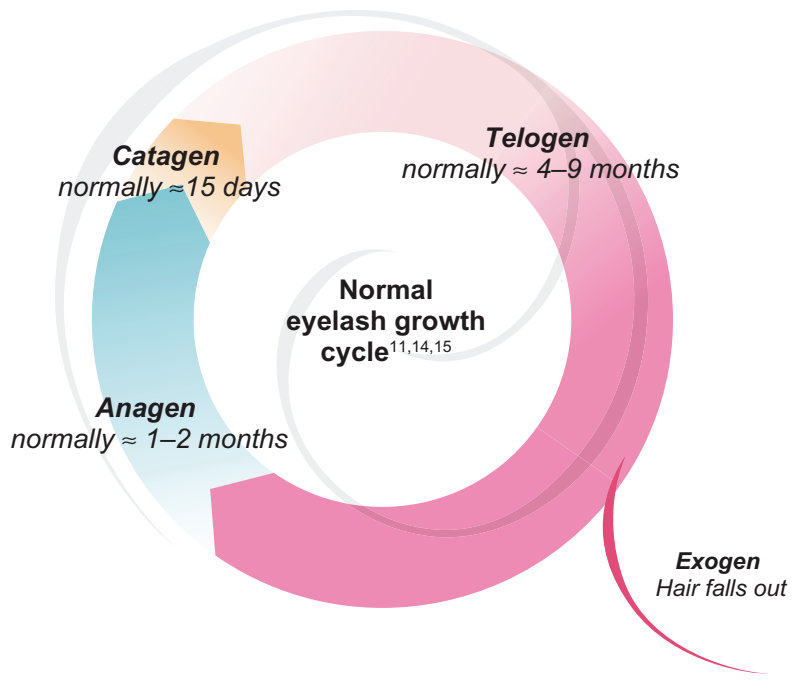

the thickest hairs on the body. ${ }^{11}$ Additionally, eyelashes are often darker than scalp hair and generally retain their pigmentation with age, making it unusual for them to turn grey. ${ }^{9,11}$ Upper eyelashes are typically arranged in two or three rows and total approximately 100 to 150 lashes per eye. ${ }^{9,10}$ Lower lashes are shorter and fewer in number than upper eyelashes. ${ }^{9,13,14}$

To understand the mechanisms of action underlying normal eyelash growth, it is important to review the current information on the eyelash hair cycle (Figure 1, data on file; Allergan, Inc.). ${ }^{7,11,14,15}$ Whereas the cycle of scalp hair has been extensively studied, few studies have been conducted on eyelashes. Traditionally thought to last approximately five to 12 months, ${ }^{11,14,15}$ new data suggest that the duration of the entire cycle may be shorter. ${ }^{13}$ The hair cycle can be divided into three main phases: anagen, catagen, and telogen. Anagen, the growth phase, is a time of rapid cell proliferation and differentiation and its duration, approximately one to two months, largely determines the length of eyelashes. ${ }^{8,11,13-16}$ Melanogenesis, and the subsequent transfer of pigment from melanocytes to medullary and cortical cells of the follicle, occurs only during anagen. ${ }^{11}$ Eyelashes grow at a rate of approximately 0.12 to $0.15 \mathrm{~mm} /$ day. ${ }^{13,15}$ This growth rate can be contrasted with the 0.3 to $0.4 \mathrm{~mm} /$ day observed in the much longer anagen phase (up to eight years) of scalp hair. ${ }^{11,12,17,18}$

During catagen, the transition phase of the hair cycle, epithelial elements of the follicle (keratinocytes) undergo programmed cell death (ie, apoptosis). ${ }^{18}$ Eyelash follicles are believed to remain in catagen for approximately 15 days. ${ }^{11}$ Following catagen, eyelashes enter telogen, the resting phase of the hair cycle. During telogen, the follicle remains dormant,

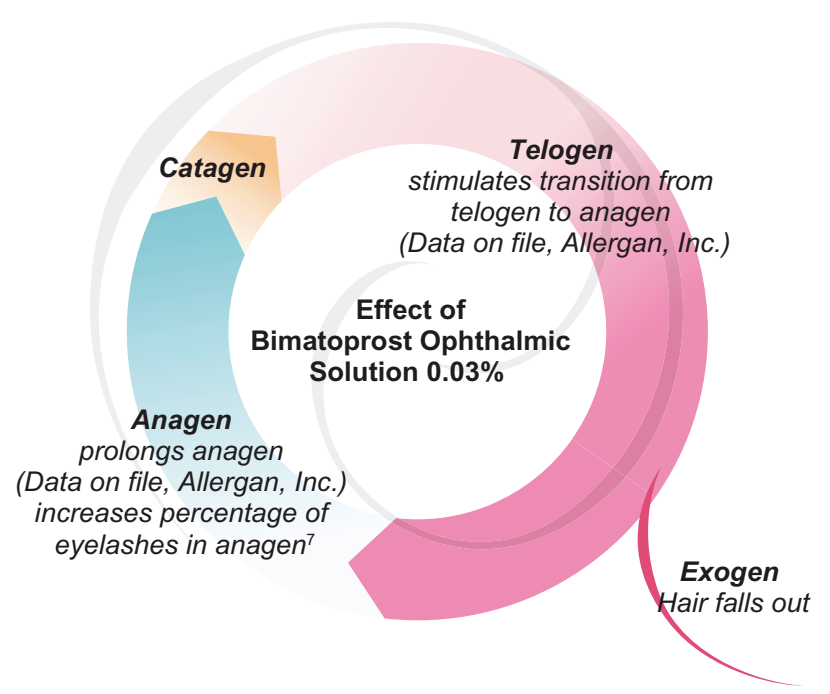

Figure I Hair cycle of normal eyelashes (left) and potential mechanisms of action of bimatoprost ophthalmic solution $0.03 \%$ on the eyelash cycle (right) (data on file, Allergan, Inc.). $7,11,14,15$ 
not exhibiting significant cell differentiation, proliferation, or apoptosis. ${ }^{16}$ The telogen phase of eyelash follicles has been reported to last from 100 days to nine months. ${ }^{11,14,15}$ The active shedding of an old eyelash occurs during the transition from telogen to anagen in a process known as exogen. ${ }^{8,16,17}$

Human hair growth is asynchronous, indicating that, at any given time, some follicles are undergoing anagen while others are in the catagen or telogen phase. ${ }^{11,12}$ Therefore, the proportion of follicles in one phase of the hair cycle is indicative of the relative duration of that phase. While approximately $41 \%$ of upper eyelid eyelash follicles are active at any given time, $84 \%$ of scalp follicles meet the same criteria. ${ }^{11,14}$ Conversely, approximately half of all eyelash follicles are in telogen compared with $5 \%$ to $15 \%$ of scalp follicles. ${ }^{11,12,14,17}$ In summary, relative to scalp hair, eyelashes have a much shorter anagen phase and a relatively longer telogen phase.

\section{Bimatoprost ophthalmic solution $\mathbf{0 . 0 3 \%}$}

Bimatoprost ophthalmic solution 0.03\% (Lumigan ${ }^{\circledR}$; Allergan, Inc.) was initially developed as an ocular antihypertensive and, in 2001, was approved for the reduction of elevated intraocular pressure (IOP) in patients with open-angle glaucoma or ocular hypertension. ${ }^{19}$ As a glaucoma treatment, bimatoprost ophthalmic solution $0.03 \%$ is administered as a drop into the affected eye once daily. It is thought to lower IOP by increasing aqueous humor outflow through the trabecular meshwork and uveoscleral pathways. ${ }^{19-23}$ The safety and effectiveness of bimatoprost ophthalmic solution $0.03 \%$ for the treatment of increased IOP has been demonstrated in numerous short- and long-term (up to four years) clinical trials. ${ }^{24-28}$ Data suggest that, presently, prostaglandin analogs such as bimatoprost ophthalmic solution $0.03 \%$ are the most efficacious antiglaucoma drugs available. ${ }^{20,29}$ A synthetic analog of prostaglandin $\mathrm{F}_{2 \alpha}$-ethanolamide or prostamide $\mathrm{F}_{2 \alpha}$, bimatoprost is known to bind to the prostamide receptor, a pharmacologically distinct receptor from prostanoid FP receptors, which are known to bind prostaglandin analogs. ${ }^{20,29}$

The recognition that bimatoprost ophthalmic solution $0.03 \%$ is capable of influencing eyelash growth was a serendipitous discovery based on observations of patients receiving the drug in trials that assessed the drug's antihypertensive properties. ${ }^{30}$ In these trials, patients commonly reported eyelash changes, which were classified as adverse events. For instance, in a pair of one-year, multicenter, randomized, double-masked, parallel-group, active-controlled trials, $42.6 \%$ of patients treated with once-daily bimatoprost (one drop instilled into each eye) experienced eyelash growth. ${ }^{28}$
Since eyelash properties were not specifically examined in the trials, such reports may have failed to capture the true incidence of these effects.

Prostaglandin analogs including latanoprost and travoprost, both used to treat glaucoma, have also been associated with eyelash changes when instilled ocularly. ${ }^{6,11,31}$ In a three-month head-to-head trial designed to directly compare the safety and efficacy of bimatoprost ophthalmic solution $0.03 \%$ and latanoprost in patients with glaucoma and ocular hypertension, the incidence of the side effect of increased eyelash growth was significantly higher in patients treated with once-daily bimatoprost than in those treated with oncedaily latanoprost. ${ }^{32}$ Whether such differences exist when the agents are applied cutaneously (ie, to the dermal margin of the eyelid) has not yet been studied. Despite proven effectiveness for the treatment of glaucoma, the safety and efficacy of dermal application of latanoprost, travoprost, and unoprostone for the purposes of eyelash growth have not been evaluated in controlled clinical trials. Furthermore, the use of these medications for aesthetic purposes is not approved by the FDA; therefore, they are not discussed in this manuscript.

Although the precise mechanisms by which bimatoprost ophthalmic solution $0.03 \%$ increases eyelash growth have not been fully elucidated, it is believed to alter the eyelash hair cycle as depicted in Figure 1 (data on file, Allergan Inc.). $7,11,14,15$ Studies conducted in mice suggest that treatment with bimatoprost results in an increased proportion of follicles being in anagen, accompanied by a simultaneous decrease in the proportion of hair follicles in telogen (data on file, Allergan, Inc.). These findings suggest that bimatoprost ophthalmic solution $0.03 \%$ stimulates the transition from telogen to anagen and prolongs the duration of anagen, thereby contributing to the increased length of eyelashes. Animal studies also demonstrate that treatment with bimatoprost ophthalmic solution $0.03 \%$ results in increased dermal papilla and hair bulb diameters among follicles undergoing early anagen. The findings are consistent with the bimatoprost-associated increased eyelash thickness also observed in mice. Increased eyelash darkness putatively results from increased melanin production. Bimatoprost-induced increases in melanogenesis do not appear to be associated with inflammatory reactions, melanocyte proliferation, or melanocyte atypia. ${ }^{33}$ Treatment with bimatoprost ophthalmic solution $0.03 \%$ does not increase the original number of eyelash follicles (data on file, Allergan, Inc.). Still, it is possible that bimatoprost ophthalmic solution $0.03 \%$ appears to increase the number of visible hairs as a result of the increased speed at which eyelashes are replaced (due to the stimulation of follicles from telogen to anagen) 
and the increased time lashes remain in the follicle (due to the prolongation of anagen and subsequent delay of exogen). Additionally, while the number of follicles remains constant, the types of hair they produce can change, such that bimatoprost may cause (previously) undetectable or less visible vellus hairs to become pigmented thicker terminal hairs..$^{19,34}$

\section{Nonbimatoprost options for eyelash enhancement}

Prior to reviewing the results from the recent pivotal trial evaluating the efficacy and safety of bimatoprost ophthalmic solution $0.03 \%$ for eyelash growth, it is important to note that women have several options available to enhance the appearance of their eyelashes. Mascara, the oldest and most commonly used option today, contains a mixture of waxes and pigments in addition to resins or petroleum distillates. ${ }^{2,35}$ It aims to temporarily darken, lengthen, and thicken eyelashes. Mascaras come in a variety of colors, formulations, and costs, and are generally accompanied by various applicators (ie, brushes). The effects are temporary and subject to smudging. Although mascara contains preservatives, a risk of microbial contamination and infection remains. ${ }^{2}$ Mascara can also cause irritation and contact dermatitis, as well as conjunctival pigmentation. ${ }^{2,35}$

Several nonmascara over-the-counter products are advertised to increase the length, fullness, and/or darkness of eyelashes. These products are sometimes marketed as "eyelash stimulators", "eyelash enhancement serums", ${ }^{37}$ or "eyelash conditioners", $38-40$ and contain various ingredients such as "proprietary peptides", ${ }^{40}$ natural extracts, ${ }^{41}$ vitamins,,${ }^{40}$ and prostaglandin analogs. ${ }^{42}$ Since cosmetic products do not require FDA approval, they have not been critically evaluated for efficacy nor has their short- or long-term safety been fully studied.

Artificial eyelashes, made from human hairs or strands of synthetic fibers, have been used by women to enhance the appearance of their eyelashes for quite some time. These lashes can be applied as a strip onto the eyelid or attached individually to existing eyelashes. ${ }^{35,43}$ When applied to individual eyelashes, the procedure can be lengthy and costly. ${ }^{43}$ Artificial eyelashes are commonly secured using glues containing methacrylate, which can cause allergic reactions in susceptible patients. ${ }^{35}$ The artificial eyelashes can remain in place from several days to several weeks, depending on the product used. ${ }^{35,43}$

Originally developed as a means of eyelash reconstruction, eyelash transplants involve the transfer of scalp follicles onto the eyelid. ${ }^{44}$ Although controversial, the procedure is also being used for aesthetic augmentation of eyelashes. ${ }^{44,45}$ Because the lashes are actually scalp hair, patients must regularly trim and curl the implanted lashes. The risks of eyelash transplantation include postoperative pain, bleeding and hematoma, scar formation, altered sensation, eyelid ptosis, dry eye, and blindness. ${ }^{44}$

\section{Efficacy of bimatoprost ophthalmic solution $\mathbf{0 . 0 3 \%}$ for eyelash growth}

When prescribed for the treatment of eyelash hypotrichosis, bimatoprost ophthalmic solution $0.03 \%$ is to be applied daily to the skin of the upper eyelid margin at the base of the eyelashes using the accompanying sterile single-use-per-eye applicators. ${ }^{7}$ Patients should be advised to apply the medication after washing their face and removing all makeup. Contact lens wearers should remove their lenses prior to application and should not reinsert them for at least 15 minutes following application. Excess solution beyond the eyelid should be absorbed with a cloth or tissue.

The efficacy of bimatoprost ophthalmic solution $0.03 \%$ to increase eyelash growth was assessed in a multicenter, double-masked, randomized, vehicle-controlled, pivotal trial. ${ }^{7,46}$ A more detailed presentation of the safety and efficacy results from the bimatoprost for eyelash growth pivotal trial is pending. Briefly, the clinical trial enrolled 278 adults who had clinician-rated "minimal" or "moderate" eyelash prominence. Participants were randomized to receive treatment with bimatoprost ophthalmic solution $0.03 \%$ $(n=137)$ or vehicle $(n=141)$ once daily for four months. The primary efficacy measure was the clinician-rated four-point Global Eyelash Assessment (GEA) scale. The GEA scale, a proven, reliable, and reproducible instrument, asks raters to evaluate a patient's overall upper eyelash prominence, focusing on the qualities of length, fullness, and darkness. The scale ranges from 1 (minimal) to 4 (very marked) and includes a photonumeric guide to assist raters. ${ }^{47}$ Response was defined as at least a 1-grade increase in GEA score. ${ }^{7,46}$ Beginning at week 8 and continuing through the end of treatment (ie, week 16), statistically significant differences in the proportion of responders favoring bimatoprost ophthalmic solution $0.03 \%$ were observed (Figure 2 ). ${ }^{7}$ By week 8 , approximately half of all patients treated with bimatoprost ophthalmic solution $0.03 \%$ experienced at least a 1 -grade increase in GEA score.

In addition to the clinician-rated GEA assessment of efficacy, changes in eyelash prominence were also evaluated using digital image analysis with quantitative measures of eyelash length, fullness/thickness, and darkness. ${ }^{7,46}$ 


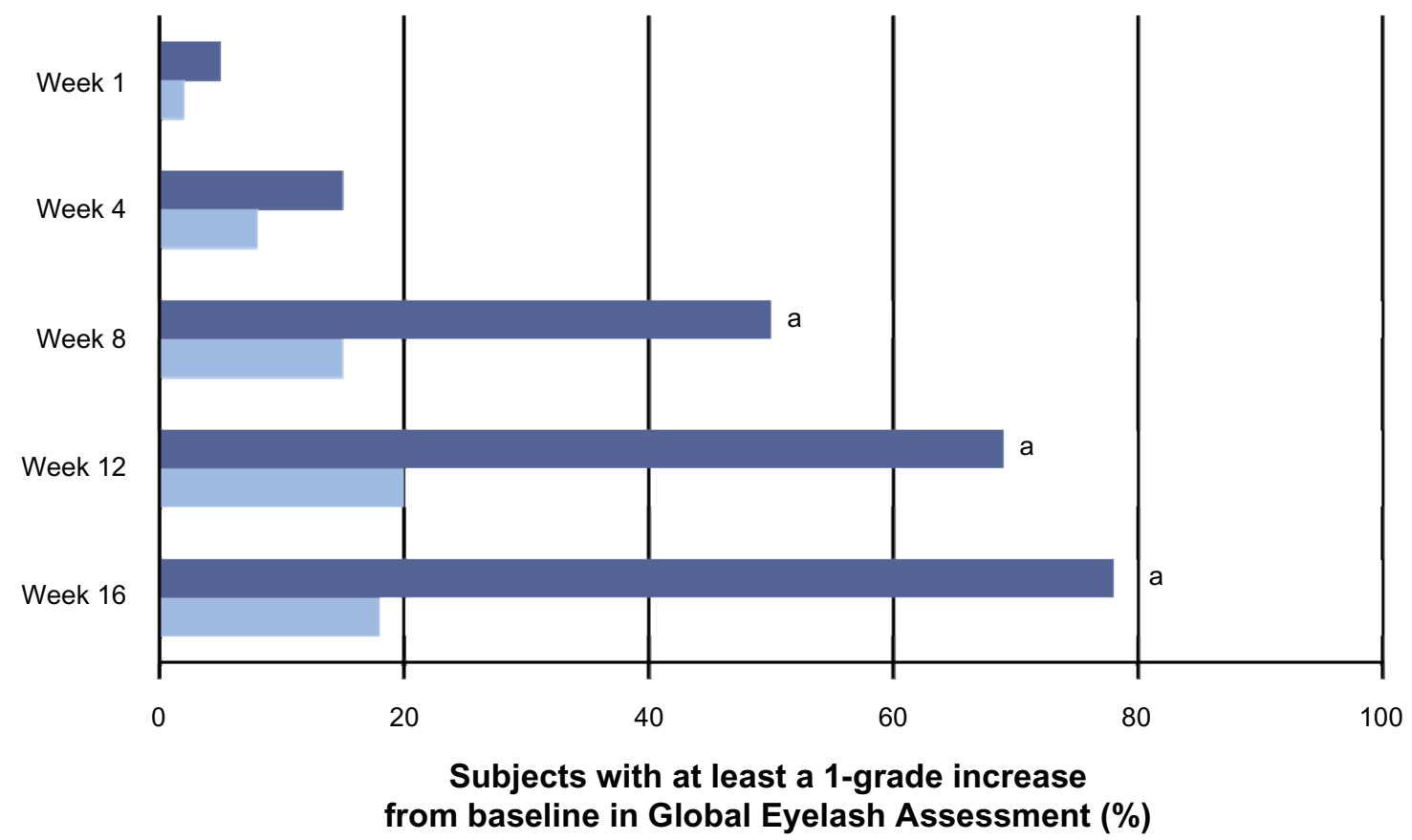

Figure 2 Efficacy of bimatoprost ophthalmic solution $0.03 \%$ as assessed by Global Eyelash Assessment. ${ }^{7}$ ap $<0.05$ vs vehicle.

Participants treated with bimatoprost ophthalmic solution $0.03 \%$ demonstrated significantly greater improvements from baseline in these three variables beginning at week 8 and continuing through the end of the treatment (ie, week 16). At the end of the treatment, bimatoprost ophthalmic solution $0.03 \%$ was associated with more than a 12 -fold greater increase in eyelash length than vehicle; a mean $1.4 \mathrm{~mm}$ increase was observed in the bimatoprost group versus $0.1 \mathrm{~mm}$ in the vehicle group. ${ }^{7}$ The percentage improvements in all three eyelash characteristics assessed by digital image analysis from baseline to week 16 were significantly greater in subjects treated with bimatoprost ophthalmic solution $0.03 \%$ than with the vehicle (Figure 3). ${ }^{7,46}$

Given the aesthetic aspects of treating hypotrichosis of the eyelashes, which can be highly personal and extend beyond objective measurements, the pivotal trial also included patient-reported outcomes (PROs) as measures of efficacy. Participants in the trial were asked to complete four questionnaires during the study including a validated 23 -item primary questionnaire, completed at baseline and all postbaseline study visits. ${ }^{48}$ Comprising three domains, the PRO measure was designed to assess the patients' static perception of the physical and subjective attributes of their eyelashes and eyelash care. The length, fullness, and overall satisfaction domain queried patients about their level of satisfaction with the physical attributes of their eyelashes. The confidence, attractiveness, and professionalism domain assessed satisfaction with subjective attributes contingent on eyelash health. Daily routine collected data on patient perceptions of the time and effort required to make eyelashes presentable. At week 16, patients treated with bimatoprost ophthalmic solution $0.03 \%$ demonstrated significant improvements from baseline in all three domains $(P<0.008)$ and all 23 items $(P<0.05)$. On a single item that asked patients to rate their overall satisfaction with their eyelashes, approximately $65 \%$ of subjects treated with bimatoprost ophthalmic solution $0.03 \%$ reported that they were "satisfied" or "very satisfied" at week 16, improved from approximately $5 \%$ at baseline. Four weeks after completing treatment, study participants were asked to rate their change in overall satisfaction with the appearance of their eyes, daily activities, and quality of life. Significant differences $(P<0.0001)$ favoring bimatoprost ophthalmic solution $0.03 \%$ were observed. For instance, $56 \%$ of subjects on bimatoprost treatment responded that their level of satisfaction was a "very great," "great," or "good" deal better than at the beginning of the study. Only $10 \%$ of patients receiving treatment with vehicle reported such levels of satisfaction.

The results of the pivotal trial indicate that bimatoprost ophthalmic solution $0.03 \%$ is an effective treatment for hypotrichosis of the eyelashes in healthy adults as measured by 
Bimatoprost ophthalmic solution $0.03 \%$

Vehicle

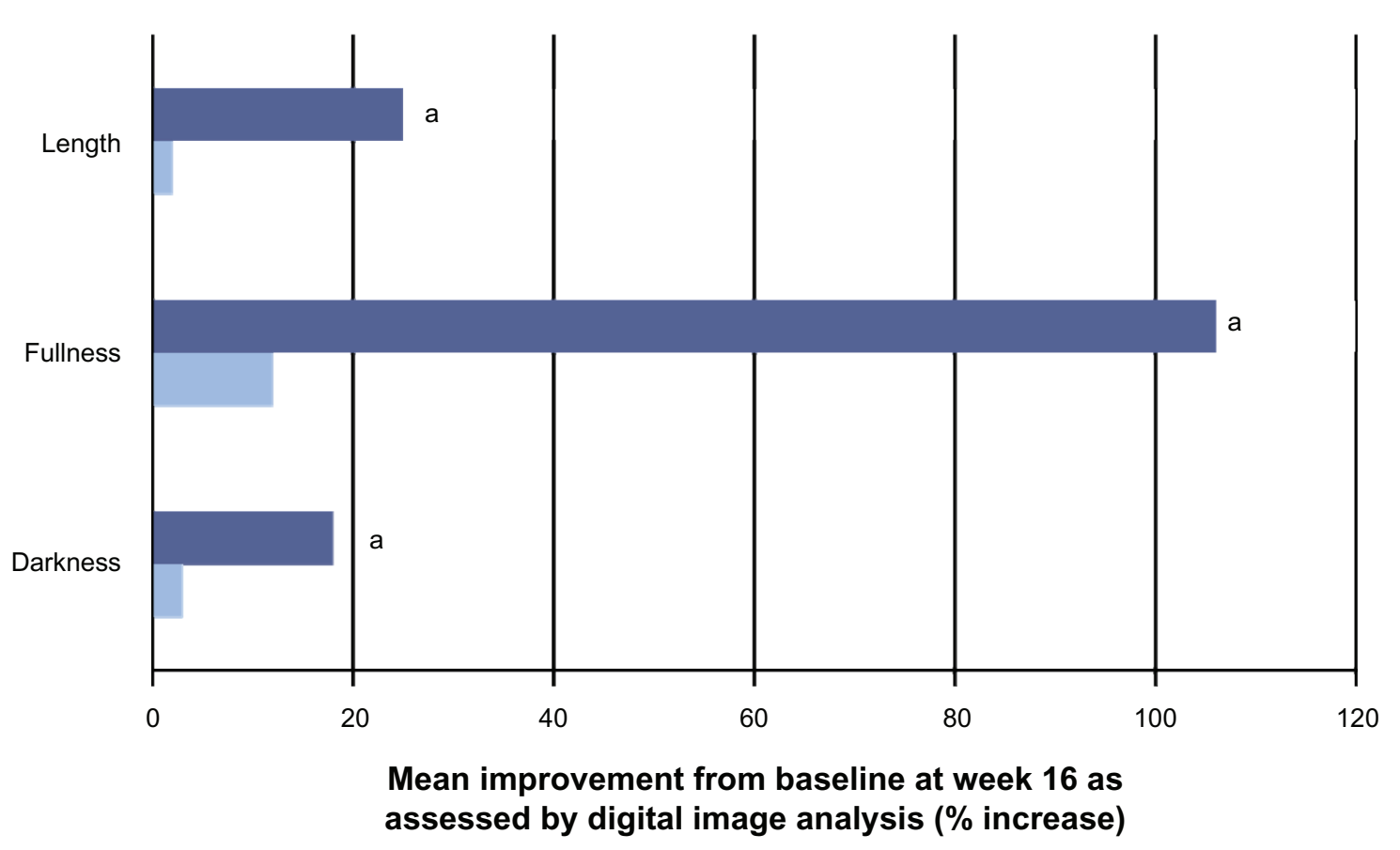

Figure 3 Efficacy of bimatoprost ophthalmic solution $0.03 \%$ as assessed by digital image analysis. ${ }^{7}$ ap $<0.05$ vs vehicle.

clinician-assessed ratings, digital image analysis, and PROs. ${ }^{46}$ While anecdotal in nature, in the author's clinical practice, once-daily bimatoprost has also demonstrated effectiveness in increasing eyelash prominence, regularly leading to noticeably longer, thicker, and darker lashes (Figure 4). The use of bimatoprost ophthalmic solution $0.03 \%$ for eyelash growth is presently only indicated in adults with hypotrichosis. The ability of bimatoprost ophthalmic solution $0.03 \%$ to influence eyelash growth in patients with eyelash loss (eg, secondary to trauma, radiation, or alopecia areata) has not been fully evaluated, although evidence suggests that some patients with alopecia of the eyelashes may benefit from treatment with bimatoprost ophthalmic solution $0.03 \%{ }^{49,50}$

\section{Safety of bimatoprost ophthalmic solution $\mathbf{0 . 0 3 \%}$ for hypotrichosis}

Evaluation of the safety of bimatoprost can draw from two data sets: the safety of ocularly instilled bimatoprost ophthalmic solution $0.03 \%$ for the treatment of ocular hypertension and the safety of dermally applied bimatoprost ophthalmic solution $0.03 \%$ for hypotrichosis of the eyelashes. Since it has a long history as a glaucoma treatment, safety data for bimatoprost ophthalmic solution $0.03 \%$ as an eyedrop are understandably more extensive than for dermal administration. As an eyedrop for the treatment of ocular hypertension, bimatoprost ophthalmic solution $0.03 \%$ has been evaluated in trials lasting up to four years. Over the course of a four-year, double-masked, randomized trial comparing bimatoprost ophthalmic solution $0.03 \%$ with timolol, eyelash growth was reported by $60.3 \%$ of patients receiving bimatoprost once daily. ${ }^{24}$ Other common adverse events related to treatment with bimatoprost ophthalmic solution $0.03 \%$ included conjunctival hyperemia (50.0\%), ocular burning (11.5\%), foreign body sensation (11.5\%), cataract (10.3\%), eye dryness $(7.7 \%)$, and eye pruritus $(6.4 \%)$. In clinical trials, bimatoprost ophthalmic solution $0.03 \%$ (as an eyedrop) was associated with pigmentation of the periocular skin in approximately $3 \%$ to $10 \%$ of patients. ${ }^{19}$

The safety profile of bimatoprost ophthalmic solution $0.03 \%$ appears considerably more favorable when applied dermally (for hypotrichosis of the eyelashes) as opposed to being instilled into the eye (for glaucoma). Differences in the exposure of ocular tissues to bimatoprost ophthalmic solution $0.03 \%$ may explain such observations. In the four-month pivotal trial assessing bimatoprost ophthalmic solution $0.03 \%$ for eyelash growth, the most common adverse events were eye pruritus, conjunctival hyperemia, skin hyperpigmentation, ocular irritation, dry eye symptoms, and erythema of the eyelid. All adverse events occurred in less than $4 \%$ of subjects receiving bimatoprost ophthalmic solution $0.03 \%$, 


\section{Baseline}
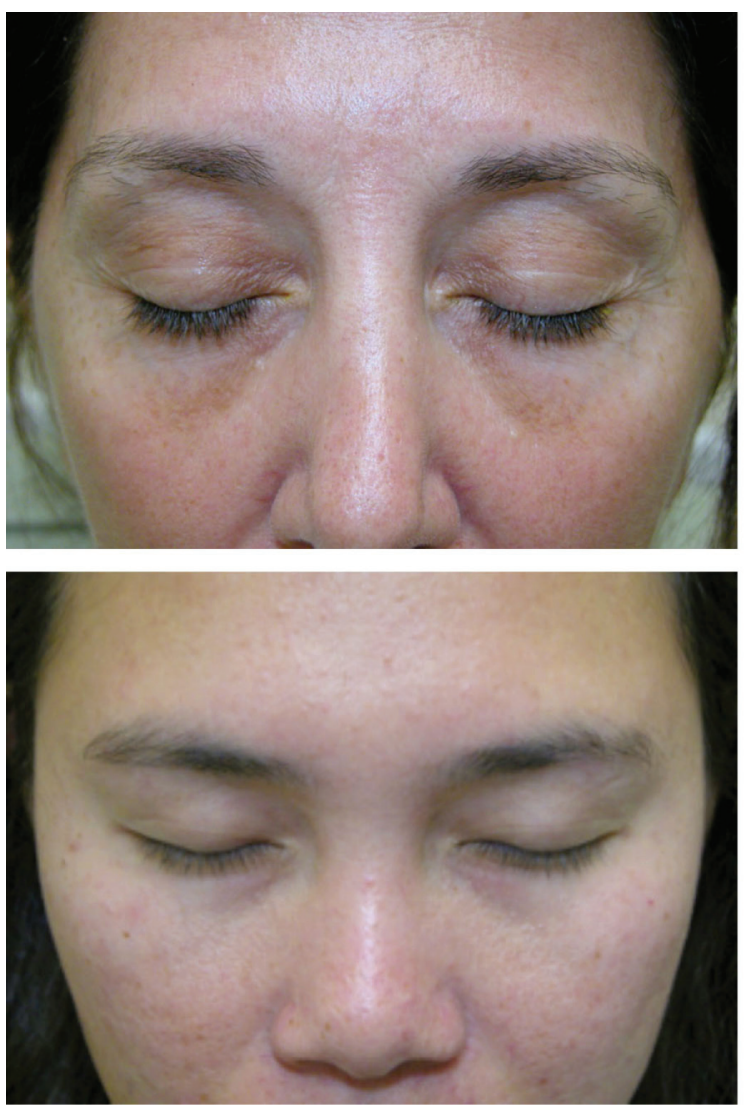

Bimatoprost-treated (12 weeks)
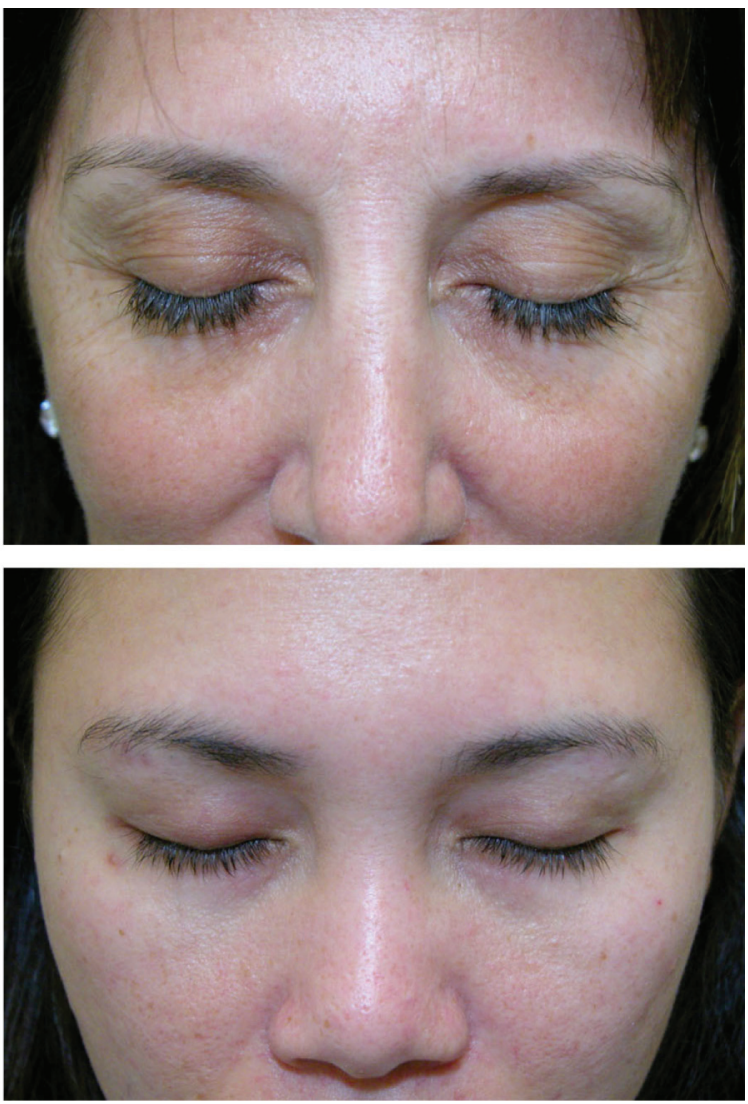

Figure 4 Sample images of patient eyelashes before and after 12 weeks of once-daily treatment with bimatoprost.

Notes: Photos courtesy of Steven Fagien, MD.

and only conjunctival hyperemia was significantly more common among subjects treated with bimatoprost ophthalmic solution $0.03 \%$ than with vehicle. ${ }^{7,46}$ Now that bimatoprost ophthalmic solution $0.03 \%$ is commercially available, larger real-life experiences with patients seems to parallel these potential "side effects" and has not been a significant cause of patient discontinuation of treatment. The incidence of these adverse events is also considerably higher when bimatoprost ophthalmic solution $0.03 \%$ is used as an eyedrop for a comparable length of therapy. For instance, in a three-month trial of once-daily treatment with bimatoprost ophthalmic solution $0.03 \%$ for glaucoma or ocular hypertension, conjunctival hyperemia, and eye pruritus were reported by $46 \%$ and $9 \%$ of patients receiving the drug, respectively. ${ }^{25}$

As an ocular antihypertensive, the incidence of increased iris pigmentation, which is believed to be a permanent change, ${ }^{51}$ associated with once-daily bimatoprost is estimated to be $1.5 \%$, with all recorded events occurring within the first year of treatment. ${ }^{24,28}$ Although this has been a significant focus of concern promulgated by the lay press and by many with little experience, no such events were observed in the clinical trial of bimatoprost ophthalmic solution $0.03 \%$ for eyelash growth. ${ }^{7,46}$ Additionally, whereas bimatoprost ophthalmic solution $0.03 \%$ instilled into the eye of patients with glaucoma significantly lowers IOP (its intended therapeutic effect), no clinically relevant changes in IOP were associated with dermal application of bimatoprost in healthy adults. ${ }^{7,46}$ While statistically significant, the mean IOP differences between the bimatoprost- and vehicle-treated cohorts during the treatment period were less than $1 \mathrm{mmHg}{ }^{46}$ The mean range of IOPs (difference of maximum and minimum IOP) during the study (baseline to week 16) was $3.7 \mathrm{mmHg}$ among vehicle-treated subjects and $3.9 \mathrm{mmHg}$ among bimatoprosttreated subjects. For perspective, normal variation in IOP over a 24-hour period among healthy subjects is approximately 7 mmHg. ${ }^{2}$

Several factors may contribute to the improved safety profile observed when bimatoprost ophthalmic solution $0.03 \%$ is applied dermally. Skin hyperpigmentation, an adverse event reversible upon discontinuation of bimatoprost, ${ }^{6,31,33}$ appears to 
be the result of drop-skin contact. ${ }^{53}$ The risk of such pigmentary changes can be minimized by reducing contact of the medication with the skin. ${ }^{53}$ Detailed instruction on safe and effective use has become commonplace in practitioner's offices that prescribe and/or dispense bimatoprost ophthalmic solution $0.03 \%$ and the proper application for eyelash growth using the supplied applicators would seemingly minimize the chance of unintended skin-bimatoprost contact. As demonstrated in an ocular splash test conducted using lissamine green dye, dermal application of bimatoprost ophthalmic solution $0.03 \%$ appears to deliver less medication to the ocular tissues than a drop instilled into the eye (Figure 5, data on file; Allergan, Inc.). Additional testing has demonstrated that, based on weight, approximately $5 \%$ of the dose of bimatoprost ophthalmic solution $0.03 \%$ on the applicator (ie, one drop) is delivered to the patient (data on file; Allergan, Inc.). Exposure of the ocular tissues to dermally applied bimatoprost ophthalmic solution $0.03 \%$ may be further decreased as a result of the skin's barrier function (data on file; Allergan, Inc.). Although evidence has demonstrated that exposure to bimatoprost ophthalmic solution $0.03 \%$ is reduced when applied dermally compared with its use as an eyedrop, the long-term safety of bimatoprost for eyelash growth has not yet been specifically evaluated in clinical trials, although such a trial is underway. ${ }^{54}$ Additionally, as the safety of bimatoprost ophthalmic solution $0.03 \%$ for the treatment of hypotrichosis of the eyelashes has only been evaluated in healthy adults, clinicians are urged to use caution when treating patients receiving concomitant treatment for elevated IOP, as well as those with intraocular inflammation, risk factors for macular edema, or aphakia. ${ }^{7}$

\section{Role in clinical practice}

Given the importance of the appearance of the eye region, and specifically the eyelashes, in influencing perceptions of beauty, it is understandable that many options to help women increase the prominence of their eyelashes have been developed. Only bimatoprost ophthalmic solution $0.03 \%$, however, is FDA-approved for the treatment of hypotrichosis of the eyelashes with proven efficacy and effectiveness. It clearly induced increased eyelash growth including improvements in length, thickness, and fullness in a controlled clinical trial. These improvements were demonstrated by objective and clinician-rated assessments as well as by participants' satisfaction with both physical and subjective attributes of their eyelashes.

While the mechanisms of action of bimatoprost ophthalmic solution $0.03 \%$ have not been fully elucidated, it appears to stimulate eyelash follicles to enter and remain in anagen, resulting in increased eyelash length. Augmented melanogenesis seems to be responsible for the increased darkness

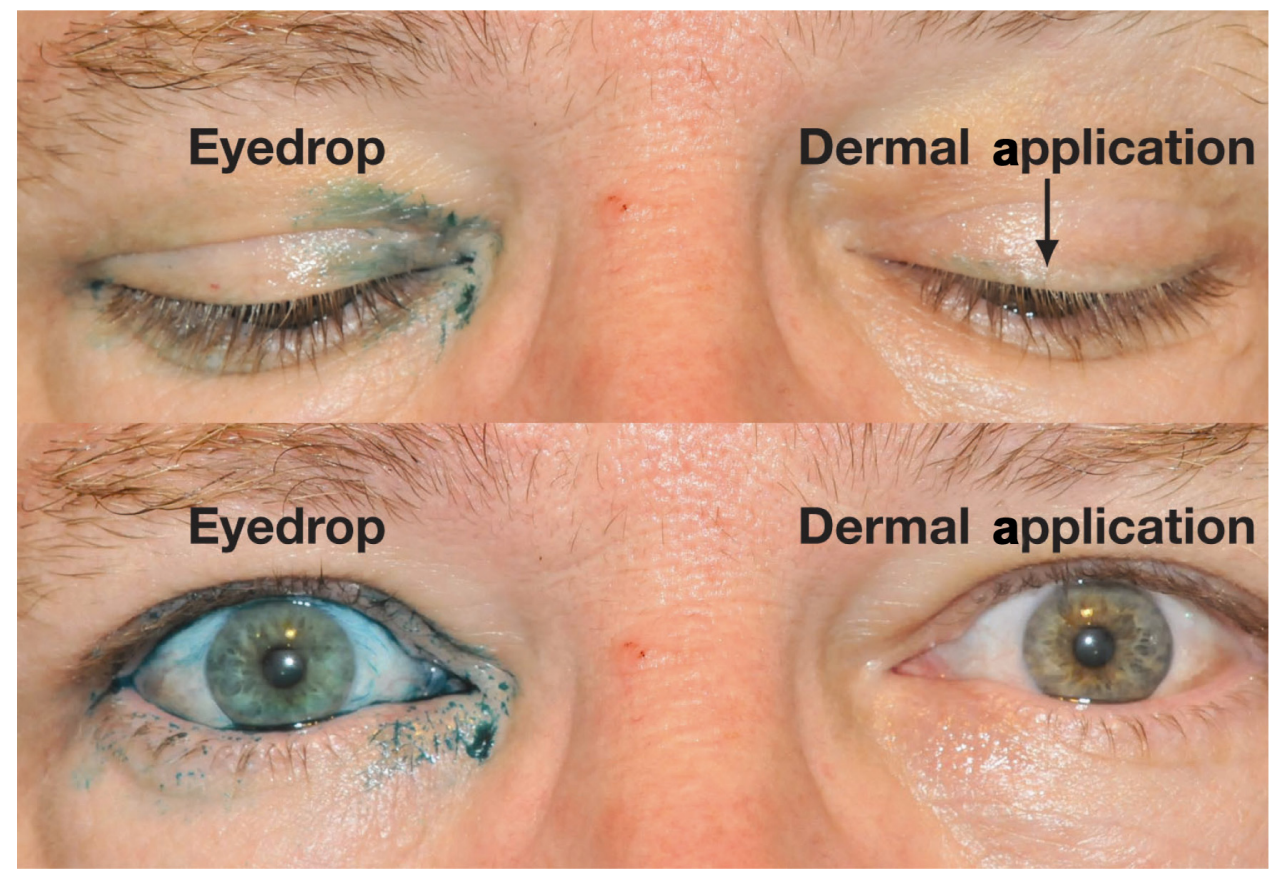

Figure 5 Example of dermal application of a tracer dye to mimic bimatoprost ophthalmic solution $0.03 \%$ to the upper eyelid margin using applicators results in the administration of a decreased dose compared with application as an eyedrop (Data on file; Allergan, Inc.). The above image was taken following administration of one drop (ie, 28-30 $\mu \mathrm{L}$ ) of lissamine green as an eyedrop (right eye [top left, bottom left]) and one drop of lissamine green transferred to a sterile brush and applied to the dermal margin of the upper eyelid (left eye [top right, bottom right]). 
of eyelashes associated with treatment, while changes in the developing hair bulb are thought to result in thicker/fuller eyelashes.

Bimatoprost ophthalmic solution $0.03 \%$ offers patients a novel method of increasing the prominence of their eyelashes. While the effects of treatment are not immediate (as with artificial eyelashes or mascara), improvements in eyelash prominence can often be observed several weeks after initiating therapy, with most patients demonstrating appreciable improvements by week 8.7,46 Importantly, these improvements appear to be sustained throughout the duration of treatment. Patients should be advised that such changes are not permanent, but instead, are believed to subside after discontinuation of treatment. ${ }^{7}$ The safety and tolerability of bimatoprost ophthalmic solution $0.03 \%$ for eyelash growth is crucial in helping to define its role in clinical practice as an aesthetic treatment. It is reassuring that the safety profile of bimatoprost ophthalmic solution $0.03 \%$ applied dermally appears even more favorable than bimatoprost ophthalmic solution $0.03 \%$ instilled ocularly, a generally safe and well tolerated treatment for increased IOP with an extensively documented safety profile originating from multiple clinical trials and long-standing postmarketing experience. Decreased exposure of the ocular tissues to the active drug may account for the reduced incidences of adverse events observed with dermal administration. Although long-term trials are needed, iridial pigmentation has not been observed in patients using bimatoprost ophthalmic solution $0.03 \%$ during the clinical trial for hypotrichosis of the eyelashes. Other mild adverse events such as conjunctival hyperemia and ocular irritation or pruritus (presumed to be a prostaglandin effect) in many seem to be self-limited and symptoms can actually improve with continuation of treatment. In both the registration trial and in clinical practice, these mild effects are a rare cause for discontinuation of therapy after patients have perceived their aesthetic improvement of lash prominence.

Finally, although only FDA-approved for application to the upper eyelid lashes, future studies might include the use of bimatoprost ophthalmic solution $0.03 \%$ for other applications including the potential for the treatment of hypotrichosis of the lower eyelid, eyebrow, and scalp. The use of this agent for the treatment of chemotherapy-induced eyelash hypotrichosis is also presently under investigation. ${ }^{55}$

Also still under speculation and yet another topic of future investigation is what alterations of the approved treatment schedule would still constitute effective treatment from the start or as maintenance once the eyelash prominence has reached a satisfactory endpoint.

\section{Conclusion}

Taken together, the available data presented herein, growing postapproval clinical experiences, and exciting future applications suggest that bimatoprost ophthalmic solution $0.03 \%$ is an effective and safe treatment in a host of applications and another therapy that appears to improve the quality of life for those patients seeking aesthetic improvement of eyelash growth and prominence.

\section{Acknowledgment}

Editorial assistance provided by Health Learning Systems, Parsippany, NJ.

\section{Disclosures}

Dr Fagien is a consultant for Allergan and BioForm, and is a clinical investigator for Allergan, Medicis, Mentor, and Dermik/Sanofi-Aventis.

\section{References}

1. Synnott A. The beauty mystique. Facial Plast Surg. 2006;22(3):163-174

2. Draelos ZD. Special considerations in eye cosmetics. Clin Dermatol. 2001;19(4):424-430.

3. Mulhern R, Fieldman G, Hussey T, Lévêque JL, Pineau P. Do cosmetics enhance female Caucasian facial attractiveness? Int J Cosmet Sci. 2003;25(4):199-205.

4. Shaikh MY, Bodla AA. Hypertrichosis of the eyelashes from prostaglandin analog use: A blessing or a bother to the patient? [Letter] J Ocul Pharmacol Ther. 2006;22(1):76-77.

5. DeMello M. Facial hair. In: DeMello M, editor. Encyclopedia of Body Adornment. Westport, CT: Greenwood Publishing Group; 2007. p. 109.

6. Holló G. The side effects of the prostaglandin analogues. Expert Opin Drug Saf. 2007;6(1):45-52.

7. Latisse [package insert]. Irvine, CA: Allergan, Inc; 2008.

8. Randall VA. Hormonal regulation of hair follicles exhibits a biological paradox. Semin Cell Dev Biol. 2007;18(2):274-285.

9. Moses RA. The eyelids. In: Moses RA, editor. Adler's Physiology of the Eye: Clinical Application. 5th ed. St. Louis, MO: C.V. Mosby Company; 1970. p. 1-16.

10. Khong JJ, Casson RJ, Huilgol SC, Selva D. Madarosis. Surv Ophthalmol. 2006;51(6):550-560.

11. Johnstone MA, Albert DM. Prostaglandin-induced hair growth. Surv Ophthalmol. 2002;47(Suppl 1):S185-S202.

12. Habif TP. Hair diseases. In: Habif TP, editor. Clinical Dermatology: A Color Guide to Diagnosis and Treatment. 4th ed. St. Louis, MO: C.V. Mosby Company; 2003.

13. Thibaut S, De Becker E, Caisey L, et al. Human eyelash characterization. Br J Dermatol. 2009 Sep 1. Epub ahead of print.

14. Elder MJ. Anatomy and physiology of eyelash follicles: Relevance to lash ablation procedures. Ophthal Plast Reconstr Surg. 1997;13(1):21-25.

15. Na JI, Kwon OS, Kim BJ, et al. Ethnic characteristics of eyelashes: A comparative analysis in Asian and Caucasian females. Br J Dermatol. 2006;155(6):1170-1176.

16. Alonso L, Fuchs E. The hair cycle. J Cell Sci. 2006;119(Pt 3):391-393.

17. Randall VA. Androgens and hair growth. Dermatol Ther. 2008; 21(5):314-328.

18. Paus R, Cotsarelis G. The biology of hair follicles. N Engl J Med. 1999;341(7):491-497.

19. Allergan, Inc. Lumigan [package insert]. Irvine, CA: Allergan, Inc; 2006. 
20. Woodward DFC, Carling RW, Cornell CL, et al. The pharmacology and therapeutic relevance of endocannabinoid derived cyclo-oxygenase (COX)-2 products. Pharmacol Ther. 2008;120(1):71-80.

21. Woodward DF, Krauss AHP, Chen J, et al. The pharmacology of bimatoprost (Lumigan ${ }^{\mathrm{TM}}$ ). Surv Ophthalmol. 2001;45(Suppl 4):S337-S345.

22. Krauss AHP, Woodward DF. Update on the mechanism of action of bimatoprost: A review and discussion of new evidence. Surv Ophthalmol. 2004;49(Suppl 1):S5-S11.

23. Brubaker RF, Schoff EO, Nau CB, Carpenter SP, Chen K, VanDenburgh AM. Effects of AGN 192024, a new ocular hypotensive agent, on aqueous dynamics. Am J Ophthalmol. 2001;131(1):19-24.

24. Williams RD, Cohen JS, Gross RL, Liu CC, Safyan E, Batoosingh AL. For the Bimatoprost Study Group. Long-term efficacy and safety of bimatoprost for intraocular pressure lowering in glaucoma and ocular hypertension: Year 4. Br J Ophthalmol. 2008;92(10):1387-1392.

25. Brandt JD, VanDenburgh AM, Chen K, Whitcup SM; For the Bimatoprost Study Group 1. Comparison of once- or twice-daily bimatoprost with twice-daily timolol in patients with elevated IOP: A 3-month clinical trial. Ophthalmology. 2001;108(6):1023-1031.

26. Noecker RS, Dirks MS, Choplin NT, Bernstein P, Batoosingh AL, Whitcup SM. For the Bimatoprost/Latanoprost Study Group. A sixmonth randomized clinical trial comparing the intraocular pressurelowering efficacy of bimatoprost and latanoprost in patients with ocular hypertension or glaucoma. Am J Ophthalmol. 2003;135(1):55-63.

27. Whitcup SM, Cantor LB, VanDenburgh AM, Chen K; for the Bimatoprost Study Group II. A randomised, double masked, multicentre clinical trial comparing bimatoprost and timolol for the treatment of glaucoma and ocular hypertension. Br J Ophthalmol. 2003;87(1):57-62.

28. Higginbotham EJ, Schuman JS, Goldberg I, et al; For the Bimatoprost Study Groups 1 and 2. One-year, randomized study comparing bimatoprost and timolol in glaucoma and ocular hypertension. Arch Ophthalmol. 2002;120(10):1286-1293.

29. Woodward DF, Liang Y, Krauss AHP. Prostamides (prostaglandinethanolamides) and their pharmacology. Br J Pharmacol. 2008;153(3): 410-419.

30. Woodson SA. Latisse ${ }^{\mathrm{TM}}$ : Empirical discovery yields treatment for sparse eyelashes. Nurs Womens Health. 2009;13(3):243-248.

31. Alm A, Grierson I, Shields MB. Side effects associated with prostaglandin analog therapy. Surv Ophthalmol. 2008;53(Suppl 1):S93-S105.

32. Gandolfi S, Simmons ST, Sturm R, Chen K, VanDenburgh AM; For the Bimatoprost Study Group 3. Three-month comparison of bimatoprost and latanoprost in patients with glaucoma and ocular hypertension. Adv Ther. 2001;18(3):110-121.

33. Kapur R, Osmanovic S, Toyran S, Edward DP. Bimatoprost-induced periocular skin hyperpigmentation: Histopathological study. Arch Ophthalmol. 2005;123(11):1541-1546.

34. Hart J, Shafranov G. Hypertrichosis of vellus hairs of the malar region after unilateral treatment with bimatoprost. Am J Ophthalmol. 2004;137(4):756-757.

35. O'Donoghue MN. Eye cosmetics. Dermatol Clin. 2000;18(4):633-639.

36. Lilash: Grow Longer and Thicker Eyelashes. Available from: http:// www.lilash.com/lilash.html. Accessed February 23, 2010.

37. neuLash Official Site - Active Eyelash Technology. Available from: http://neulash.com/index.htm. Accessed February 23, 2010.

38. RevitaLash Product Catalog. Available from: http://www.revitalash. com/product-catalog.php. Accessed February 23, 2010.
39. MD Lash Factor Eyelash Conditioner. Available from: http://www. mdlashfactor.com. Accessed February 23, 2010.

40. Marini Lash Eyelash Conditioner. Available from: http://www. myjanmarini.com/pc/Marini-Lash ${ }^{\mathrm{TM}}$-Eyelash-Conditioner-12p50. htm. Accessed February 23, 2010.

41. Actifirm ActiLash. Available from: http://shop.actifirm.com/products/ actifirm-actilash. Accessed February 23, 2010.

42. Choy I, Lin S. Eyelash enhancement properties of topical dechloro ethylcloprostenolamide. J Cosmet Laser Ther. 2008;10(2): $110-113$.

43. Maxwell A. Eyes open wide with these lash extensions. Available from: http://www.usatoday.com/life/lifestyle/2006-03-27-eyelashes_x.htm. Accessed February 23, 2010

44. Straub PM. Replacing facial hair. Facial Plast Surg. 2008;24(4): 446-452.

45. Hernández-Zendejas G, Guerrerosantos J. Eyelash reconstruction and aesthetic augmentation with strip composite sideburn graft. Plast Reconstr Surg. 1998;101(7):1978-1980.

46. Smith S, Fagien S, Somogyi C, Whitcup SM, Beddingfield FC. Eyelash growth in subjects treated with bimatoprost ophthalmic solution $0.03 \%$; A multicenter, randomized, double-masked, vehicle-controlled, parallel study. Poster presented at: American Academy of Dermatology's 67th Annual Meeting; March 6-9, 2009; San Francisco, CA.

47. Yoelin S, Wu J, Somogyi C, Beddingfield FCI. Inter-rater and intra-rater reliability of the Global Eyelash Assessment scale for assessment of overall eyelash prominence. Poster presented at: Skin Disease Education Foundation's 33rd Annual Hawaii Dermatology Seminar; February 7-13, 2009; Maui, HI.

48. Fagien S, Walt JG, Carruthers J, et al. Patient-reported outcomes of bimatoprost for eyelash growth: Results from a randomized, doublemasked, vehicle-controlled, parallel group study. Poster presented at: American Society for Aesthetic Plastic Surgery Aesthetic Meeting; May 2-7, 2009; Las Vegas, NV.

49. Zaheri S, Hughes B. Successful use of bimatoprost in the treatment of alopecia of the eyelashes. Clin Exp Dermatol. 2009 Nov 19. Epub ahead of print.

50. Ochoa BE, Sah D, Wang G, Stamper R, Price VH. Instilled bimatoprost ophthalmic solution in patients with eyelash alopecia areata. $J$ Am Acad Dermatol. 2009;61(3):530-532.

51. Stjernschantz JW, Albert DM, Hu D-N, Drago F, Wistrand PJ. Mechanism and clinical significance of prostaglandin-induced iris pigmentation. Surv Ophthalmol. 2002;47(Suppl 1):S162-S175.

52. Liu JH, Sit AJ, Weinreb RN. Variation of 24-hour intraocular pressure in healthy individuals: Right eye versus left eye. Ophthalmology. 2005;112(10):1670-1675

53. Centofanti M, Oddone F, Chimenti S, Tanga L, Citarella L, Manni G. Prevention of dermatologic side effects of bimatoprost $0.03 \%$ topical therapy. Am J Ophthalmol. 2006;142(6):1059-1060.

54. Safety and efficacy study of bimatoprost to treat hypotrichosis of the eyelashes after application to the eyelid margin. Available from: http://clinicaltrials.gov/ct2/show/NCT00907426. Accessed February 23, 2010.

55. Bimatoprost ophthalmic solution in increasing eyebrow and eyelash growth in patients who have undergone chemotherapy for breast cancer and in healthy participants. Available from: http://clinicaltrials.gov/ct2/ show/NCT00999557. Accessed February 23, 2010.
Clinical, Cosmetic and Investigational Dermatology

\section{Publish your work in this journal}

Clinical, Cosmetic and Investigational Dermatology is an international, peer-reviewed, open access, online journal that focuses on the latest clinical and experimental research in all aspects of skin disease and cosmetic interventions. All areas of dermatology will be covered; contributions will be welcomed from all clinicians and

\section{Dovepress}

basic science researchers globally. This journal is indexed on CAS The manuscript management system is completely online and includes a very quick and fair peer-review system, which is all easy to use. Visit http://www.dovepress.com/testimonials.php to read real quotes from published authors. 